\title{
0.04 degree-per-hour MEMS disk resonator gyroscope with high-quality factor (510 k) and long decaying time constant (74.9s)
}

Qingsong Li (1)', Dingbang Xiao ${ }^{1,2}$, Xin Zhou', Yi Xu', Ming Zhuo', Zhanqiang Hou ${ }^{1,2}$, Kaixuan He ${ }^{3,4}$, Yongmeng Zhang ${ }^{1}$ and Xuezhong $\mathrm{Wu}^{1,2}$

\begin{abstract}
The disk resonator gyroscope is an attractive candidate for high-performance MEMS gyroscopes. This gyroscope consists of a sensor and readout electronics, and the characteristics of the sensor directly determine the performance. For the sensor, a high-quality factor and long decaying time constant are the most important characteristics required to achieve high performance. We report a disk resonator gyroscope with a measured quality factor of $510 \mathrm{k}$ and decaying time constant of $74.9 \mathrm{~s}$, which is a record for MEMS silicon disk resonator gyroscopes, to the best of our knowledge. To improve the quality factor of the DRG, the quality factor improvement mechanism is first analyzed, and based on this mechanism two stiffness-mass decoupled methods, i.e., spoke length distribution optimization and lumped mass configuration design, are proposed and demonstrated. A disk resonator gyroscope prototype is fabricated based on these design strategies, and the sensor itself shows an angle random walk as low as $0.001 \% \sqrt{ } \mathrm{h}$, demonstrating true potential to achieve navigation-grade performance. The gyroscope with readout electronics shows an angle random walk of $0.01 \% \mathrm{Vh}$ and a bias instability of $0.04 \% \mathrm{~h}$ at room temperature without compensation, revealing that the performance of the gyroscope is severely limited by the readout electronics, which should be further improved. We expect that the quality factor improvement methods can be used in the design of other MEMS gyroscopes and that the newly designed DRG can be further improved to achieve navigation-grade performances for high-end industrial, transportation, aerospace, and automotive applications.
\end{abstract}

\section{Introduction}

High-performance microelectromechanical system (MEMS) gyroscopes are in wide demand in high-end industrial, transportation, aerospace, and automotive applications. Considerable research has been conducted on MEMS Coriolis vibratory gyroscopes (CVGs), such as disk $^{1-13}$, bulk acoustic wave ${ }^{14}$, quad mass ${ }^{15}$, and microscale 3D wineglass gyroscopes ${ }^{16,17}$. Among these, the disk resonator gyroscope (DRG) is an attractive candidate for

\footnotetext{
Correspondence: Dingbang Xiao (dingbangxiao@nudt.edu.cn)

${ }^{1}$ College of Intelligence Science, National University of Defense Technology, Changsha 410073, China

${ }^{2}$ Laboratory of Science and Technology on Integrated Logistics Support, National University of Defense Technology, Changsha 410073, China

Full list of author information is available at the end of the article.
}

high-performance MEMS gyroscopes due to its inherent mode matching, high thermal stability, low anchor loss, and abundant electrodes. The first $0.01 \%$ navigationgrade MEMS DRG with active temperature control and compensation was previously reported ${ }^{1}$ and demonstrated the potential of DRGs for use in high-performance MEMS gyroscopes.

A high-quality factor $(\mathrm{Q})$, long decaying time constant $(\tau)$, high symmetry of damping, and mode matching are the essential attributes of high-performance $\mathrm{CVGs}^{1,16,17}$. In MEMS CVGs, a quad mass gyroscope (QMG) with a $Q$ value $>1.7$ million and $\tau$ longer than $328 \mathrm{~s}$, has been reported $^{15}$. A bulk acoustic wave (BAW) gyroscope with $Q$ up to 1.38 million has been measured at a $2.745 \mathrm{MHz}$ center frequency ${ }^{14}$. A $Q$ factor as high as 4.45 million and 
a decaying time constant longer than $259 \mathrm{~s}$ have been demonstrated on a fused silica birdbath shell resonator ${ }^{17}$. For DRGs, the state-of-art of the reported highest $Q$ was 0.358 million, and the longest decaying time constant was $38.5 \mathrm{~s}$ in our previous report ${ }^{18}$; these values severely limit the potential performance improvement. Therefore, it is necessary to further improve the $Q$ and $\tau$ of DRGs, for which $\tau=Q / \pi f_{0}$ and $f_{0}$ is the resonant frequency.

The quality factor is a measure of the energy dissipation in vibrating resonators. Several energy dissipation mechanisms exist, including air damping, surface loss, support loss, electrical damping, and thermoelastic damping (TED $)^{19-23}$. In DRGs encapsulated in highvacuum packages and working in elliptical modes, TED has been identified as the major energy dissipation mechanism and imposes an upper limit on the attainable $Q^{8,24}$. Therefore, thermoelastic quality factor $\left(Q_{\mathrm{TED}}\right)$ improvement is the main target in optimization of DRGs. The TED can be separated into two categories: materialdependent and geometry-dependent components ${ }^{25-27}$. For MEMS DRGs, which are always manufactured with silicon, the material-dependent component has been fixed, and thus high $Q_{\text {TED }}$ mechanical design becomes a highly significant issue. Certain examples of $Q_{T E D}$ improvement exist via innovative structural design or structural optimization for DRGs. Reference studies ${ }^{8,12}$ have presented a method for $Q_{\text {TED }}$ improvement by lengthening the spokes in DRGs and manipulating the heat flux paths in the resonator. Our previous work proposed a method for improving the $Q_{\mathrm{TED}}$ of DRGs by optimizing the ring thickness distribution ${ }^{28-30}$.

However, in most research, the $Q_{\mathrm{TED}}$ improvement mechanisms were not clearly explained, and the $Q$ and $\tau$ of DRGs can only be slightly improved. In this paper, we present the $Q_{\text {TED }}$ improvement mechanism in the DRG, and according to this mechanism, two novel stiffnessdecoupled methods are proposed for $Q$ and $\tau$ improvement. The first method is stiffness distribution optimization achieved by spoke length distribution (SLD) optimization, in which the spoke lengths on different layers of the DRG are designed to be varied, instead of identical in the traditional DRGs, and the optimum SLD is obtained using the particle swarm optimization (PSO) method. The other method is mass distribution optimization achieved by the lumped mass configuration, in which the lumped masses are hung on the ring frame to increase the effective mass while maintaining the stability of the effective stiffness. The effectiveness of the two methods is demonstrated with the finite element method (FEM) and the experimental results. The newly designed DRG based on these design strategies is fabricated and characterized. The $Q$ and $\tau$ are tested as $510 \mathrm{k}$ and $74.9 \mathrm{~s}$, respectively, which are the records for MEMS DRGs. The theoretical angle random walk (ARW) of the DRG sensor itself is as low as $0.001 \% \sqrt{ } \mathrm{h}$, showing a potential to achieve navigation-grade performance. In contrast, the ARW and bias instability of the gyroscope are tested as $0.01 \% \sqrt{\mathrm{h}}$ and $0.04^{\circ} / \mathrm{h}$, respectively, showing that the noise and instability of the gyroscope are severely dominated by the readout electronics. Improvement of the readout electronics could further reduce the ARW and instability of the gyroscope for high-end applications. Furthermore, the $Q$ and $\tau$ improvement methods proposed in this paper can be broadly used in the optimization of MEMS-vibrating gyroscopes and other resonators.

\section{Results}

\section{Model and operation of the DRG}

The traditional DRG shown in Fig. 1a consists of a disk resonator, 16 outer electrodes, and layers of inner electrodes. The disk resonator is composed of several concentric ring frames interconnected through eight spokes and a single central anchor. The device thickness, ring number, outer radius, anchor radius, anchor radius to outer radius ratio (AOR), and ring width are defined as $T$, $N, R, r, \delta(r / R)$, and $h$, respectively. The disk resonator is excited at the $n=2$ elliptical driving mode, and the Coriolis force is detected at the degenerate sensing mode, which is located geometrically $45^{\circ}$ apart from the driving mode, as shown in Fig. 1b. Ideally, the two modes have an identical natural frequency but indistinguishable mode shapes.,

Due to the complexity of the relationships between the geometrical parameters and the DRG characteristics, the effects of the geometrical parameters on the DRG $Q_{T E D}$ and $f_{0}$ are investigated using FEM. The results are shown in Fig. 2. It can be observed that the ring width and outer radius are the main factors affecting the $Q_{\mathrm{TED}}$ in the DRG, whereas the ring number and AOR have slight influences. Therefore, for the traditional DRGs with identical ring width and ring gaps, to improve the $Q_{T E D}$, the ring width should be as small as possible and the outer radius should be as large as possible. However, the ring width is limited by the fabrication capability, and the outer radius is limited by the device size. Thus for the traditional DRGs, $Q_{\text {TED }}$ improvement will come to an end only by changing the basic geometrical parameters $h, N, R$, and $\delta$. In this paper, two novel methods are presented to break the limit.

\section{$Q_{\text {TED }}$ improvement mechanism in DRGs}

The temperature deviation and heat flow in the vibrating DRG are shown in Fig. 3a, illustrating that the main thermoelastic dissipation originates from the in-plane bending deformation of the ring frames and spokes in the DRG. The disk resonator can be viewed as the combination of thin beams with different length and shapes. Thus, it can be assumed that the $f-Q$ relationship of DRGs can be expressed with the widely applied Zener 

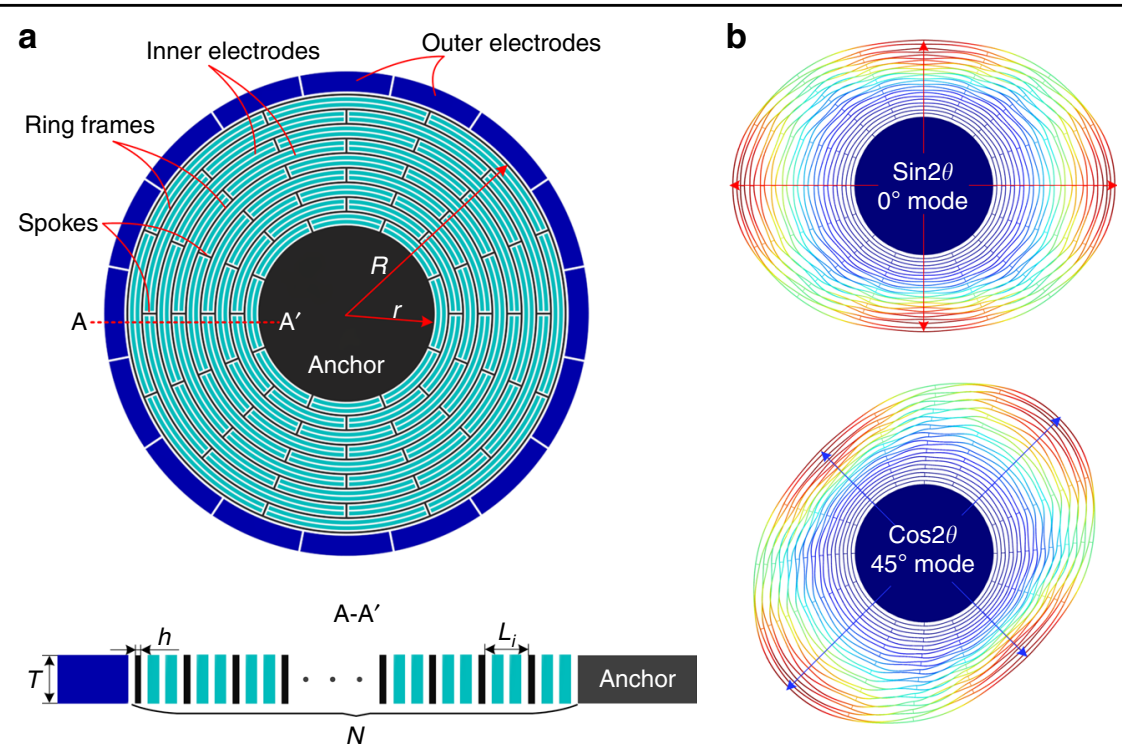

Fig. 1 a Schematic view of the traditional disk resonator gyroscope. $\mathbf{b}$ Working modes of the DRG

one-dimensional TED theoretical model: ${ }^{25,26}$

$$
\begin{aligned}
Q_{T E D} & =\frac{2 C_{v}}{E \alpha^{2} T_{0}} \cdot \frac{1+\left(f_{0} / f_{\text {Relax }}\right)^{2}}{2\left(f_{0} / f_{\text {Relax }}\right)} \\
f_{\text {Relax }} & =\frac{\pi k}{2 h^{2} C_{v}}
\end{aligned}
$$

Herein, $f_{0}$ and $f_{\text {Relax }}$ are resonant frequency and thermal relaxation frequency, respectively; $E$ is the Young's modulus; $\alpha$ is the linear coefficient of thermal expansion; $T_{0}$ is the equilibrium temperature of the beam; $C_{\mathrm{v}}$ is the specific heat per unit volume; $k$ is the thermal conductivity of the beam; and $h$ is the beam width in the flexural direction. The Zener curve and the simulated results for the different geometrical parameters presented in Fig. 2 are compared in Fig. 3b, showing that the simulated $Q$ values are slightly lower than the theoretical $Q$ due to neglect of the out-of-plane energy dissipation. However, in the design of DRGs, slight errors can be neglected compared with the other errors. Thus the Zener 1D TED model can be used in analysis of the TED in DRGs.

The Zener curve shows that the maximum dissipation occurs at the Debye peak, where $f_{0}=f_{\text {Relax }}$. In the lowfrequency range $f_{0} \ll f_{\text {Relax }}$, the strain rate is reduced and the temperature gradient is consequently reduced, leading to low levels of energy dissipation, and thus the system is considered isothermal. In the high-frequency range $f_{0} \gg f_{\text {Relax }}$, notably low levels of energy are dissipated, similar to the low-frequency range, and the system is adiabatic. Because most of the DRGs operate in the lowfrequency range, it can be observed that the key to improving $Q_{\text {TED }}$ in DRGs is to minimize the normalized frequency $f_{0} / f_{\text {Relax }}$. For the DRG with a certain ring width, $f_{\text {Relax }}$ is fixed, and thus the most effective way to improve $Q_{\text {TED }}$ is to decrease its resonant frequency $f_{0}$.

The DRG with $N$ rings can be treated as the combination of $N$ resonators coupled in a series of different stiffness and masses, as depicted in Fig. 3c, d where $m_{i}$ is the mass of the rings and $k_{i}$ is the effective stiffness affected by the ring width, ring radius, and spoke lengths. Improvement of $Q$ by changing the basic geometrical parameters reaches a limit because the stiffness and mass of the DRG are coupled with each other. This paper proposes two novel methods to decouple the stiffness and mass of the DRG and further improve the $Q$ factor of DRGs. One method is SLD optimization, in which the spoke lengths are changed to primarily affect the stiffness distribution of the DRG and further change the resonant frequency, as depicted in Fig. 3c. The other method is lumped mass configuration, in which the lumped masses are hung on the ring frame to primarily affect the mass distribution of the DRG and also change the resonant frequency, as depicted in Fig. 3d. This paper aims to find the optimum SLD and the lumped mass configuration to further improve the quality factor of the DRG.

\section{SLD optimization}

To simplify the analysis, a nine-ring DRG model is used. The length of the spokes on the $i$ th layer of the ring is defined as $L_{i}$. This optimization is applied under the condition that the other structural parameters $R, r, h$, and $N$ are fixed. Thus, the total sum of the spoke lengths should be constant:

$$
\sum_{i=1}^{n} L_{i}=R-r-N h=\text { Sum }
$$



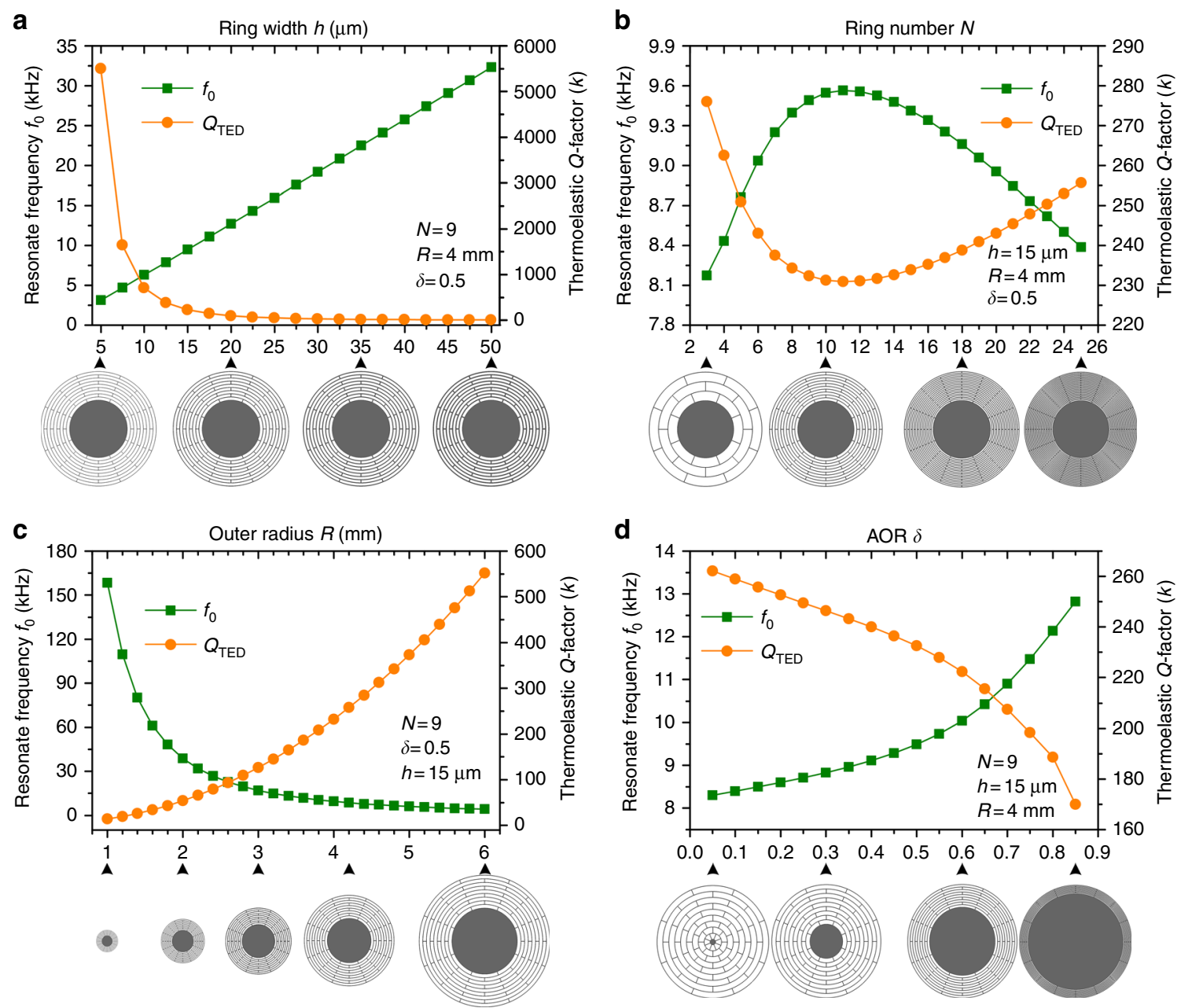

Fig. 2 a Effects of geometrical parameters on the resonant frequency and QTED of the DRGs. Ring width varying from $5 \mu \mathrm{m}$ to $50 \mu \mathrm{m}$, $\mathbf{b}$ ring numbers varying from 3 to 25 , c outer radius varying from $1 \mathrm{~mm}$ to $6 \mathrm{~mm}$, and $\mathbf{d}$ outer radius to anchor radius ratio varying from 0.05 to 0.85

Additionally, the spoke lengths should be limited in a range from $a$ to $b$ :

$$
a \leq L_{i} \leq b \quad(i=1,2,3 \ldots n)
$$

Herein, the ring number $N$ is 9 , and $R, r, h, a$, and $b$ are set as $4 \mathrm{~mm}, 1.9 \mathrm{~mm}, 12 \mu \mathrm{m}, 0.2 \mathrm{~mm}$, and $0.6 \mathrm{~mm}$, respectively, according to the actual requirement of the device size and the fabrication capability.

Stochastic evolutionary algorithms are proposed and widely applied to solve multidimensional design optimization problems. For example, the genetic algorithm (GA) was used in multidisciplinary system design of microgyroscopes ${ }^{31}$, ant colony optimization (ACO) was applied for structural topology optimization ${ }^{32}$, and PSO was chosen for structural optimization of MEMS resonators for $Q$ factor improvement ${ }^{33}$. In this paper, the PSO method $^{34,35}$ is used to find the optimum SLD for the highest $Q_{\text {TED }}$ because of its simple formula and powerful function. The SLD can be expressed as $L=\left(L_{1}, L_{2}, L_{3}, L_{4}\right.$, $L_{5}, L_{6}, L_{7}, L_{8}$, and $\left.L_{9}\right)$. Each distribution can be viewed as a nine-dimensional location of a particle, and $Q_{\mathrm{TED}}$ is an implicit function of $L$ that can be treated as the fitness value in PSO. A total of ten particles and 50 iterations are selected as a trade-off between computing time and tolerance. The steps in the PSO process are listed as follows: (a) randomly initialize the positions and velocities of all particles in the problem space shown in Equations (4) and (5); (b) calculate the fitness value $\left(Q_{\mathrm{TED}}\right)$ of each particle using COMSOL Multiphysics; (c) for every particle, compare the current fitness value with the pbest (the best fitness this particle has achieved so far) and update pbest with the largest value; (d) for every particle, compare the current fitness with the gbest (the best fitness ever searched by all of the particles in the previous iterations) and update gbest with the largest value; (e) update the velocity of the particle according to the 

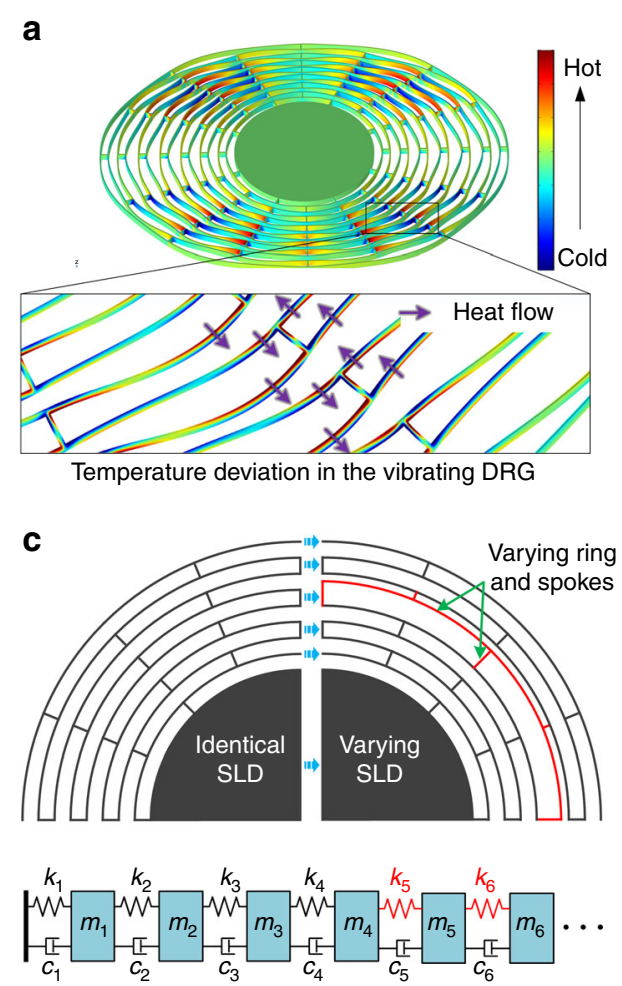

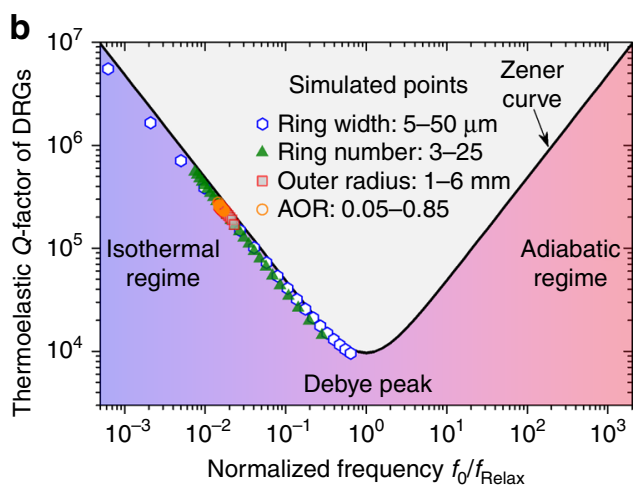

d
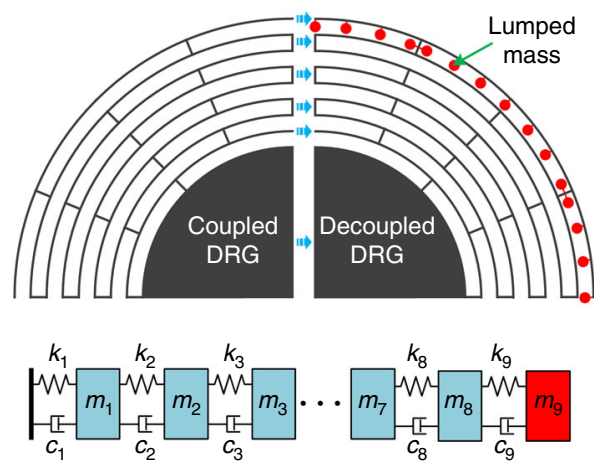

Fig. 3 a Temperature deviation of the full device and heat flow in the beams at the working modes. $\mathbf{b}$ Zener curve and comparison of the TED in DRGs with different geometrical parameters obtained by the Zener model and COMSOL simulation. $\mathbf{c}$ Schematic of the stiffness distribution optimization method: spoke length distribution optimization. $\mathbf{d}$ Schematic of the mass distribution optimization method: lumped mass configuration

following:

$$
\begin{aligned}
v_{j}^{k+1}= & \omega v_{j}^{k}+c_{1} \operatorname{Rand}(0,1)\left(\text { pbest }-x_{j}^{k}\right) \\
& +c_{2} \operatorname{Rand}^{\prime}(0,1)\left(\text { gbest }-x_{j}^{k}\right)
\end{aligned}
$$

where $v_{j}^{k}$ and $x_{j}^{k}$ are the velocity and position of the $j$ th particle at the $k$ th iteration, $\operatorname{Rand}(0,1)$ and $\operatorname{Rand}^{\prime}(0,1)$ are two separately generated random numbers within $[0,1], \omega$ is the inertia weight, $c_{1}$ and $c_{2}$ are acceleration coefficients, and in this investigation, we set $c_{1}, c_{2}$, and $\omega$ to $1.49,1.49$, and 0.729 , respectively; (f) update the position of the particle according to the following

$$
x_{j}^{k+1}=x_{j}^{k}+v_{j}^{k+1}
$$

(g) Loop to step (b). The iteration is terminated when sufficiently good fitness or the maximum number of iterations is achieved.

The PSO processes of the natural frequency $f_{0}$ and $Q_{\text {TED }}$ of the nine-ring DRG are shown in Fig. 4a, b. Five trails are shown, and trails 1 and 3 converge to the largest optimum. The SLD optimization processes in trail 1 are shown in Fig. 4c, and the optimized SLD is shown in Fig. $4 \mathrm{~d}$. It can be observed that the global optimum is achieved when the lengths of spokes 1-4 and 6-9 reach the lower boundary of $0.2 \mathrm{~mm}$, whereas the length of spoke 5 is the residual of the constant Sum and the other spoke lengths, $0.392 \mathrm{~mm}$. Additionally, the global optimum $Q_{\text {TED }}$ is $\sim 490 \mathrm{k}$, which is a $25 \%$ improvement compared with the $Q_{\text {TED }}$ of the traditional DRG with the identical spoke length (392 k).

\section{Lumped mass configuration}

In the lumped mass configuration, the masses are hung on the ring frames and spokes with a single beam. The lumped masses can greatly change the masses in the DRGs while slightly affecting the effective stiffness. As shown in Fig. 5a, with the increase of the lumped masses layers from 0 to 4 , the effective mass shows an 8.4-fold improvement, whereas the effective stiffness only changes by up to $36 \%$. Thus the resonant frequency can be greatly decreased, while the thermal relaxation frequency remains nearly constant. The thermoelastic dissipation in the lumped masses can be neglected because their elastic deformation is notably small. Thus, the Zener 1D TED model is still suitable for the stiffness-mass decoupled DRG, as shown in Fig. 5b. From Fig. 5b, it can also be observed that with increase of the lumped mass layers, $Q_{\text {TED }}$ is improved. However, the improvement ratio 

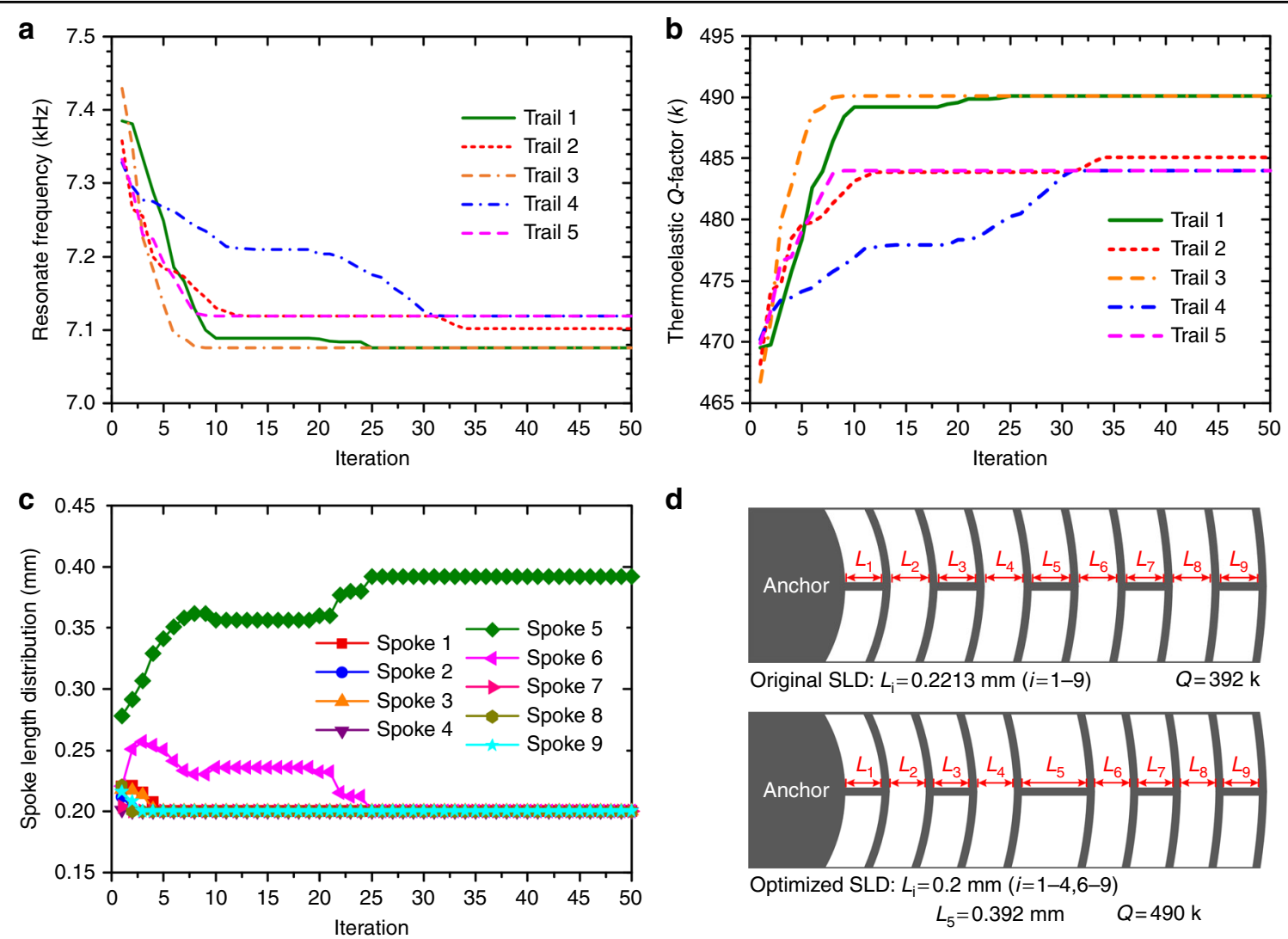

d
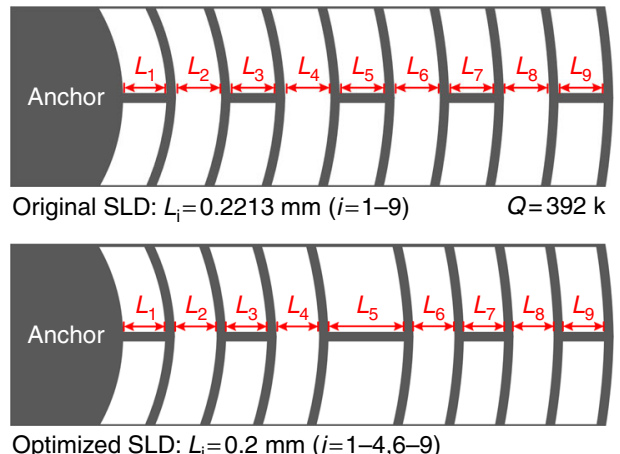

Optimized SLD: $L_{\mathrm{i}}=0.2 \mathrm{~mm}(i=1-4,6-9)$

$L_{5}=0.392 \mathrm{~mm} \quad Q=490 \mathrm{k}$

Fig. 4 a, b Plots of the resonant frequency $f_{0}$ and $Q_{\text {TED }}$ of the DRG at each iteration in the PSO progress. $\mathbf{c}$ Plot of the spoke length distributions at each iteration in trail 1. d Schematic view of the spoke length distribution in the traditional nine-ring DRG and the DRG with optimized SLD
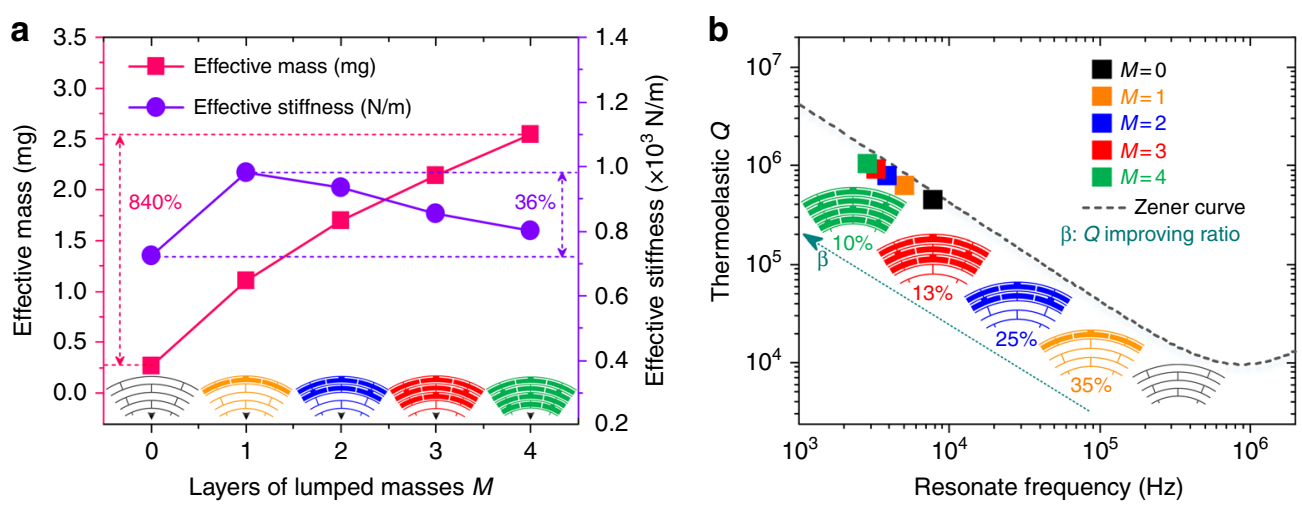

Fig. $\mathbf{5}$ a Effects of the layers of the lumped masses on the effective mass and stiffness of the DRG. $\mathbf{b}$ Effects of the layers of the lumped masses on the resonant frequency and $Q_{\text {TED }}$ of the DRG

decreases gradually from $35 \%$ to $10 \%$ because the lumped masses hanging on the outer rings have a larger contribution to the effective mass improvement than the lumped masses hanging on the inner rings. The layers of lumped masses are set to four as a trade-off between the $Q$ factor and the number of inner electrodes. The inner electrodes can be used to improve the capabilities of driving, sensing, and tuning of the DRG.

\section{Model and prototype of the new DRG}

The schematics of the resonators in the traditional DRG (DRG I), the DRG with SLD optimization (DRG II), and the final design using both optimization methods (DRG III) are shown in Fig. 6a. The resonant frequency $Q_{T E D}$ and the decaying time constant $\tau$ of the three types of DRGs are compared in Fig. 6b. This figure shows that with use of the SLD optimization, $Q$ and $\tau$ of the DRG are 


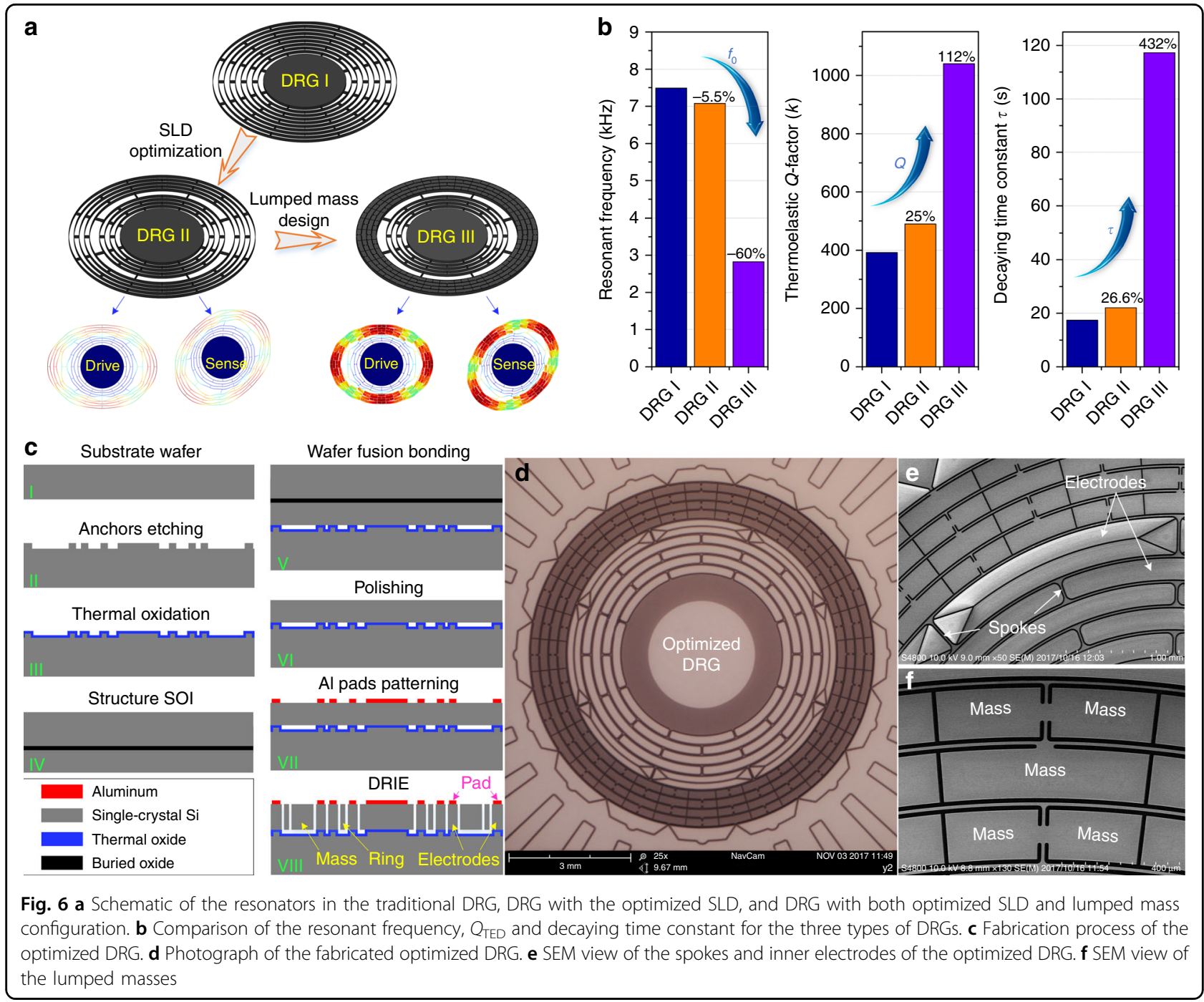

improved by $\sim 25 \%$ and $\sim 26.6 \%$, respectively. Furthermore, using the stiffness-mass decoupled design, $Q$ and $\tau$ of the DRG are greatly improved by $112 \%$ and $432 \%$, respectively. These improvements can directly reduce the bias instability and mechanical noise of the gyroscope. This disk resonator is fabricated using wafer fusion bonding and deep reactive ion etching (DRIE) processes. Detailed introductions to the fabrication processes are supplied in the Materials and Methods section. The schematics of the fabrication processes and images of the fabricated DRG are shown in Fig. 6c-f.

\section{Gyroscope performance characterization}

To characterize the properties of the optimized sensor, the frequency response and ring-down tests are implemented. Using the frequency response analyzer (NF FRA5087), the frequency responses of the driving and sensing modes of the DRG are obtained as shown in Fig. $7 \mathrm{a}$, showing that the initial frequency split between the two modes is approximately $1.338 \mathrm{~Hz}\left(\Delta f / f_{0}=\right.$
$600 \mathrm{ppm})$. For the high $Q$ resonator, the frequencydomain $Q$ measurement is often difficult and inaccurate due to nonlinear effects. Therefore, the ring-down technique is used in this work for $Q$ factor measurement. The ring-down testing results are shown in Fig. 7b, c. The figure shows that $Q$ of the optimized DRG reaches $510 \mathrm{k}$ and the decaying time constant reaches $74.9 \mathrm{~s}$. To the best of our knowledge, this result presents the highest $Q$ and longest decaying time constant demonstrated in MEMS silicon DRGs to date. This result also demonstrates that the optimization methods proposed in this paper are effective for $Q$ and $\tau$ improvement in DRGs. A discrepancy between the measured and simulated quality factors reveals that the other damping factors in DRGs can no longer be neglected if the $Q$ is greater than $500 \mathrm{k}$. Because the air pressure is sufficiently low, air damping can be neglected. Thus, residual damping mainly originates from the support damping, surface damping, and electrical damping, which should be reduced in future research. 

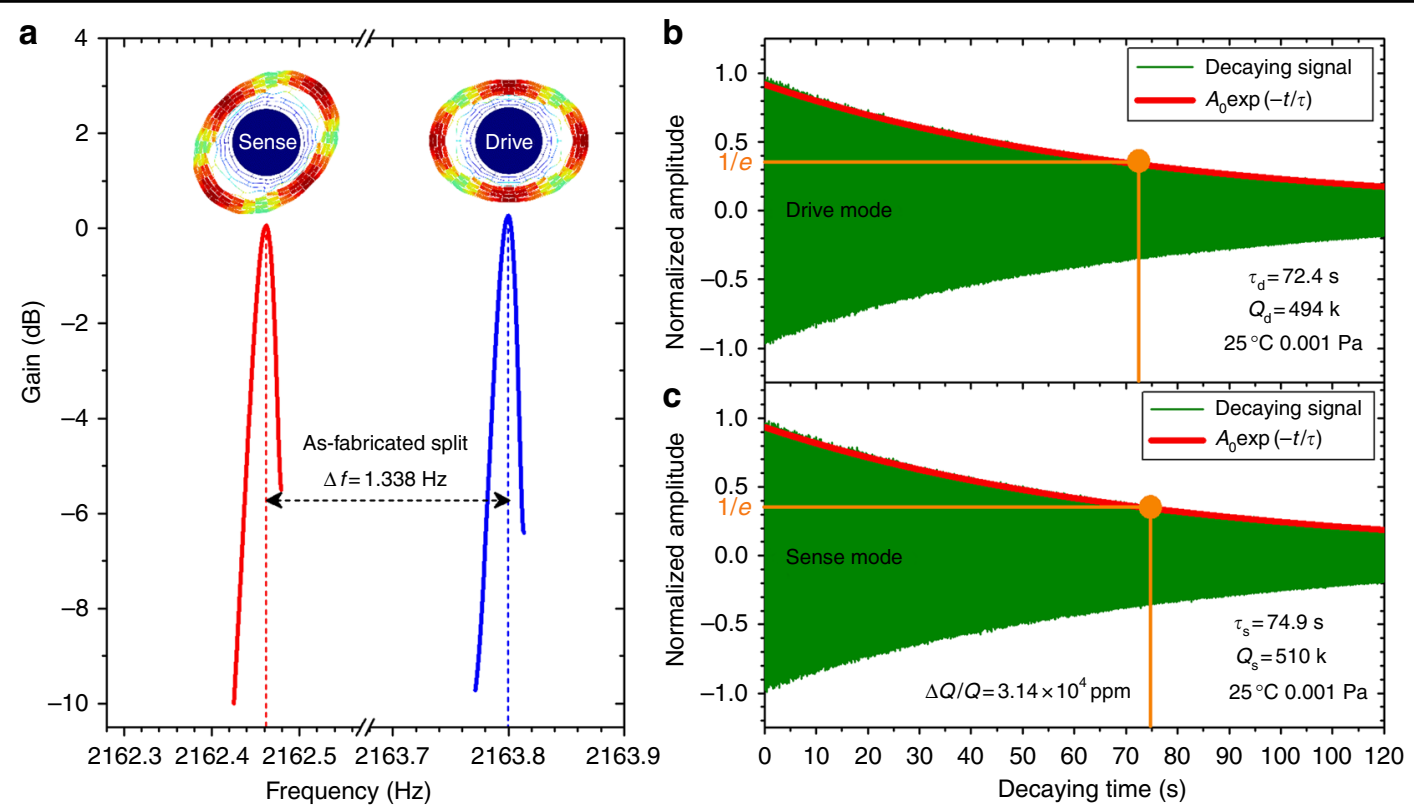

Fig. 7 a Frequency response of the driving and sensing modes in the optimized DRG. $\mathbf{b}$, c Ring-down testing results of the drive mode and sense mode for the DRG

The schematic of the readout circuit is shown in Fig. 8a. The gyroscope is driven to fixed displacement with the phase-locked loop (PLL) and proportional integral derivative (PID) controller. The drive/sense axes misalignment is tuned with the quadrature error control loop. The Coriolis force is detected using the analog-closed loop based on a modified PID controller, which can also be replaced with a digital control strategy based on the principle of a sigma-delta modulator $(\Sigma \Delta \mathrm{M})$ for higher performance ${ }^{36}$. The schematic of the readout circuit is shown in Fig. 8a, in which the driving control loop, sensing control loop, and quadrature error control loop operate simultaneously. In the mode-matched gyroscopes, the frequency split greatly affects the mechanical sensitivity and bias stability of the gyroscope. For high $Q$ resonators, the mechanical bandwidth is so narrow that mode matching cannot be accurately distinguished with FRA. Therefore, in this paper, the mode-matching method relies on monitoring of the Coriolis channel output in response to the quadrature electrode dither ${ }^{37,38}$. This method takes advantage of the fact that the quadrature leaks into the rate output only if a frequency mismatch occurs between the drive and sense mode, and the gyroscope output is proportional to the frequency mismatch. The $2 \mathrm{~Hz}$ sine dither signal is added to the axistuning voltage, and the various frequency-tuning voltages are applied on the four inner electrodes aligned with the high-frequency mode. The gyroscope output signals at several frequency-tuning voltages are shown in Fig. 8b. It can be noted that the amplitude and phase of the output are affected by the frequency-tuning voltages. With more accurate measurement, the mode-matching voltage is obtained as $30.9 \mathrm{~V}$, where the output amplitude is zero, as shown in Fig. 8c.

In the readout circuit, $\pm 12 \mathrm{~V}$ power voltage is used, and the signal is differentially output such that the maximum output voltage is only $\pm 24 \mathrm{~V}$. The angular inputs are set as $\pm 1^{\circ} / \mathrm{s}, \pm 2^{\circ} / \mathrm{s}, \pm 3^{\circ} / \mathrm{s}, \pm 4^{\circ} / \mathrm{s}, \pm 5^{\circ} / \mathrm{s}, \pm 6^{\circ} / \mathrm{s}, \pm 7^{\circ} / \mathrm{s}, \pm 8^{\circ} / \mathrm{s}, \pm 9^{\circ} / \mathrm{s}$, $\pm 10^{\circ} / \mathrm{s}, \pm 20^{\circ} / \mathrm{s}, \pm 50^{\circ} / \mathrm{s}$, and $\pm 100^{\circ} / \mathrm{s}$. The testing points in $\pm 10 \%$ are denser than those out of $\pm 10 \%$ s because in our application, the range of $\pm 10 \%$ is often used. As shown in Fig. $8 \mathrm{~d}$, the gyroscope output maintains a linear response to the rate inputs, showing a scale factor of $0.2138 \mathrm{~V} /(\% / \mathrm{s})$ and a zero offset of $0.0206^{\circ} \mathrm{s}$ due to the anisodamping of $3.14 \times 10^{4} \mathrm{ppm}$. The bias stability performance is tested at room temperature, and the gyroscope output is recorded over $7 \mathrm{~h}$. The optimized DRG shows a peak-peak noise of $4.4^{\circ} / \mathrm{h}$ at the sampling frequency of $1 \mathrm{~Hz}$, as shown in Fig. 8e. Furthermore, the effective displacement $x_{0}$ of the DRG can be derived as $2 \mu \mathrm{m}$ by detecting the vibrating voltage in the capacitance readout circuit of the driving loop. The effective mass $m_{\text {eff }}$ can be simulated as $2.54 \mathrm{mg}$. With the measured $Q$ and resonant frequency $f_{0}$, the

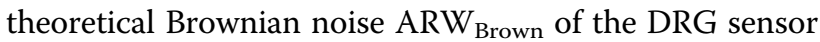
can be calculated as low as $0.001^{\circ} / \sqrt{ } \mathrm{h}$ (green trace in Fig. 8f) according to the following:

$$
A R W_{\text {Brown }}=\frac{1}{2 A_{g} x_{0}} \sqrt{\frac{k_{B} T}{2 \pi m_{\text {eff }} f_{0} Q}} \times\left(\frac{180}{\pi} \times 60^{\circ} / \sqrt{h}\right)
$$



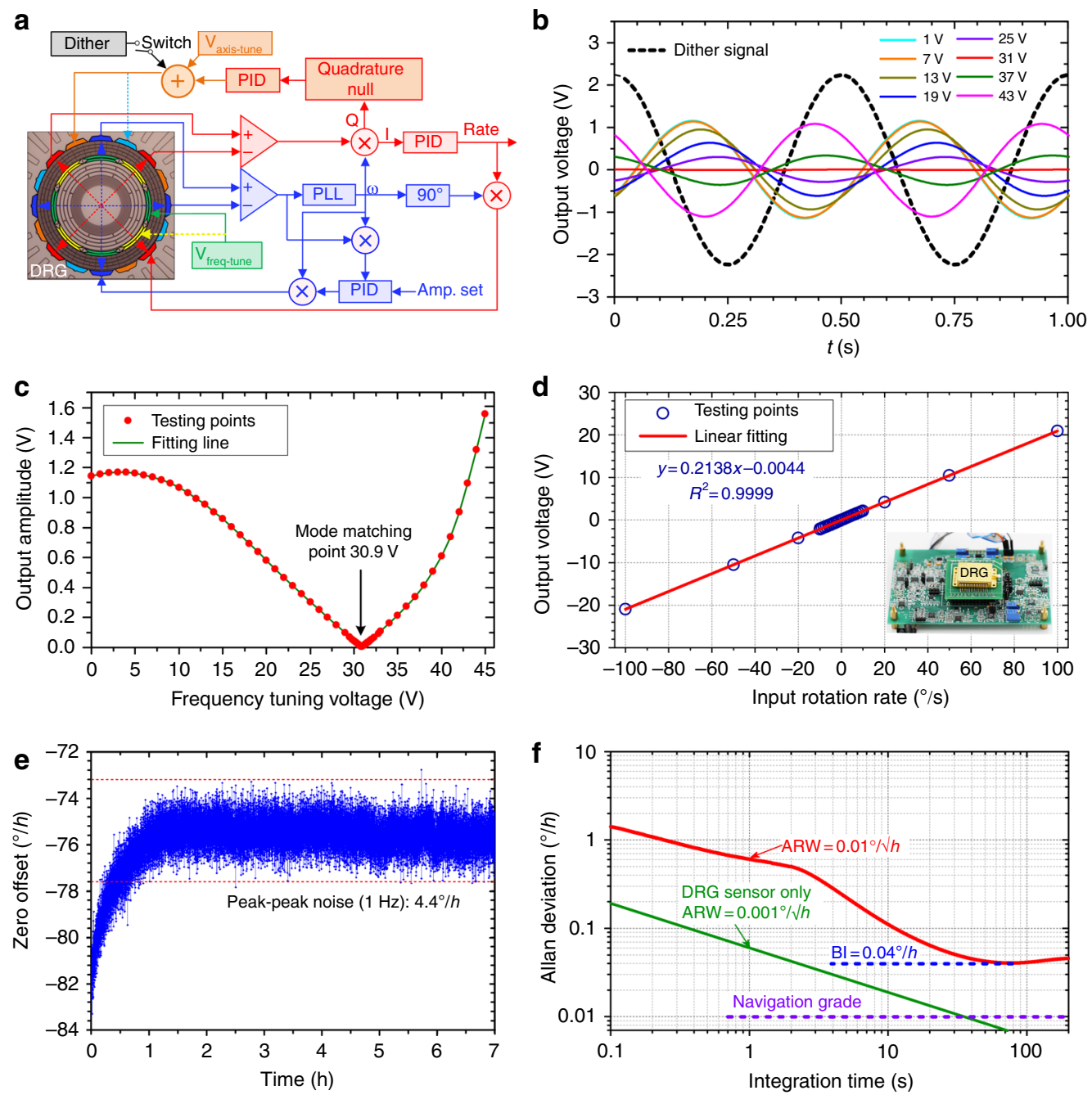

Fig. 8 a Readout circuit of the optimized DRG. b Gyroscope output signal and $\mathbf{c}$ output amplitude due to the quadrature electrode dither at various frequency-tuning voltages ranging from 0 to $45 \mathrm{~V}$. d Output response of the DRG to rotation rate range from $-100^{\circ} / \mathrm{s}$ to $100^{\circ} / \mathrm{s}$. e Bias offset test of the DRG for $7 \mathrm{~h}$ at room temperature. $\mathbf{f}$ Allan deviation plot of the optimized DRG and the sensor only

Herein, $A_{\mathrm{g}}$ is the angular gain, $k_{B}$ is the Boltzmann's constant, and $T$ is the absolute ambient temperature. Furthermore, the ARW and bias instability are tested as $0.01^{\circ} / \sqrt{\mathrm{h}}$ and $0.04^{\circ} / \mathrm{h}$ at room temperature without any compensation or postprocessing of data. This result shows that the ARW and bias instability of the gyroscope are dominated by the noise and drift of the readout electronics and that they must be further improved.

\section{Discussion}

The performances of the reported MEMS DRGs and the new DRG in this paper are listed in Table 1. It can be observed that the optimized DRG presented in this work has the highest $Q$ and the longest decaying time constant, and therefore the DRG has a lower ARW and a bias instability close to that of the navigation grade. However, the readout electronics severely limit the performance of the gyroscope. In our future work, the readout electronics will be improved to decrease the noise and drift.

We present the quality factor improvement mechanism in DRGs and propose two novel methods for the quality factor improvement. With these two novel methods, the quality factor and decaying time constant of the newly designed DRG are greatly improved, giving it the potential to achieve navigation-grade performance. These quality factor improvement methods can be further applied in the optimization of other micromechanical resonators to improve their performances for high-end markets.

\section{Materials and methods}

In this paper, COMSOL Multiphysics is chosen for simulation of $Q_{\text {TED }}$ and the resonant frequency of the DRG. In the simulation, the material properties are given as follows: $E=170 \mathrm{GPa}$, density $\rho=2329 \mathrm{~kg} / \mathrm{m}^{3}$, 


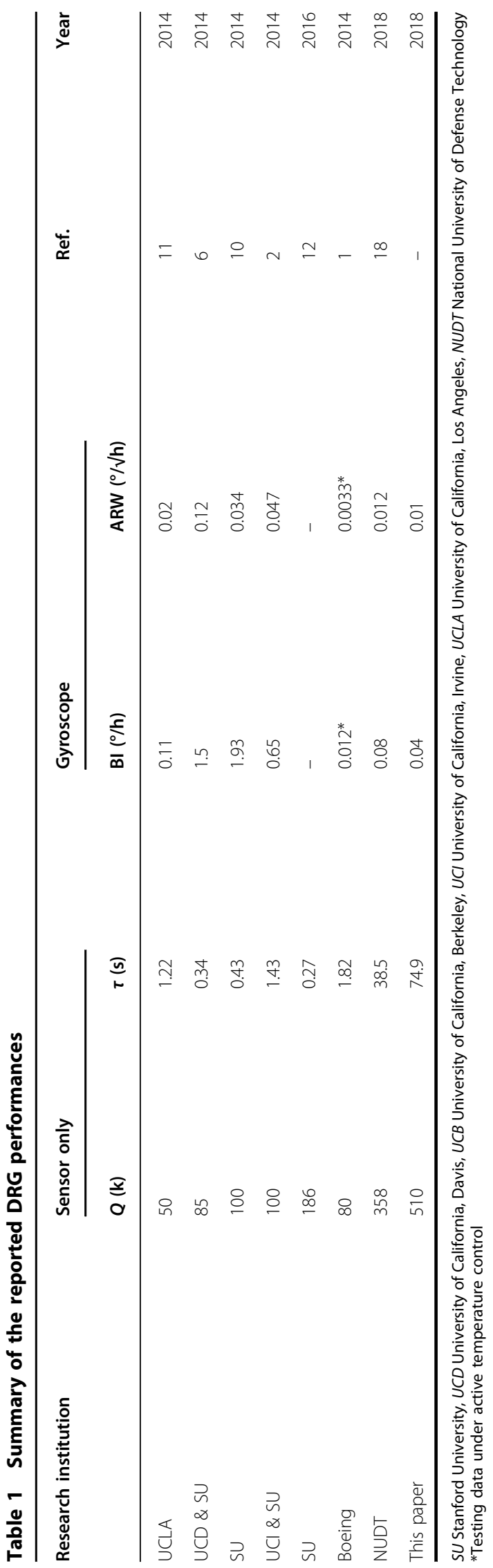

$\alpha=2.6 \times 10^{-6} \mathrm{~K}^{-1}, \quad C_{v}=1.63 \times 10^{6} \mathrm{Jm}^{-3} \mathrm{~K}^{-1}, \quad k=$ $130 \mathrm{Wm}^{-1} \mathrm{~K}^{-1}$, and $T_{0}=293.15 \mathrm{~K}$.

This disk resonator is fabricated using wafer fusion bonding and a DRIE process, as shown in Fig. 6c. A P-type $500-\mu \mathrm{m}$ (111) single-crystal silicon wafer is used as the substrate, and an SOI wafer with a $150-\mu \mathrm{m}$ (111) singlecrystal silicon device layer is used in resonator fabrication. First, the substrate is etched via DRIE for $10 \mu \mathrm{m}$ to form the anchors corresponding to the electrodes. Second, thermal oxidation technology is used to generate the thermal oxide layer on the substrate. Using wafer fusion bonding technology, the SOI wafer and the substrate are bonded together, and the handle wafer is moved with chemical and mechanical polishing. Finally, the aluminum wire bonding pads are patterned, and DRIE technology is applied for resonator and electrode fabrication.

\section{Acknowledgements}

This work was supported by the National Natural Science Foundation of China under Grant 51575521 and the 2017 Huxiang Provincial Scholar Program. This support is gratefully acknowledged.

\section{Author details}

${ }^{1}$ College of Intelligence Science, National University of Defense Technology, Changsha 410073, China. ${ }^{2}$ Laboratory of Science and Technology on Integrated Logistics Support, National University of Defense Technology, Changsha 410073, China. ${ }^{3}$ State Key Laboratory of ASIC and System, School of Microelectronics, Fudan University, Shanghai 200433, China. ${ }^{4}$ East China Institute of Photo-Electron IC, Bengbu 233042, China

\section{Author contributions}

Qingsong Li proposed the spoke length distribution optimization method and wrote the manuscript, Dingbang Xiao and Xin Zhou proposed the stiffnessmass decoupled method and designed the disk resonator gyroscope, Yi Xu conducted the FEM simulation, Ming Zhuo conducted performance testing of the gyroscope, Kaixuan He fabricated the device, Zhanqiang Hou finished the vacuum package of the DRG, Yongmeng Zhang designed the readout circuit, and Xuezhong Wu assisted in writing and correction of the manuscript.

Conflict of interest

The authors declare that they have no conflict of interest.

Received: 2 June 2018 Revised: 6 August 2018 Accepted: 4 September 2018 Published online: 19 November 2018

\section{References}

1. Challoner, A. D., Ge, H. H. \& Liu, J. Y. Boeing disc resonator gyroscope. In 2014 IEEE/ION Position, Location and Navigation Symposium (PLANS 2014); 5-8 May 2014; 504-514 (Monterey, CA, 2014).

2. Senkal, D. et al. 100k Q-factor toroidal ring gyroscope implemented in waferlevel epitaxial silicon encapsulation process. In IEEE 27th International Conference on Micro Electro Mechanical Systems (MEMS 2014); 26-30 Jan 2014, 24-27 (2014)

3. Su, T. H. et al. Silicon MEMS disk resonator gyroscope with an integrated CMOS analog front-end. leee. Sens. J. 14, 3426-3432 (2014).

4. Nitzan, S. H. et al. Self-induced parametric amplification arising from nonlinear elastic coupling in a micromechanical resonating disk gyroscope. Sci. Rep. $\mathbf{5}$, 9036 (2015).

5. Ahn, C. H. et al. Encapsulated disk resonator gyroscope with differential internal electrodes. In IEEE 29th International Conference on Micro Electro Mechanical Systems (MEMS 2016); 24-28 Jan 2016; 962-965 (Shanghai, 2016).

6. Nitzan, S. et al. Impact of gyroscope operation above the critical bifurcation threshold on scale factor and bias instability. In IEEE 27th International 
Conference on Micro Electro Mechanical Systems (MEMS 2014); 26-30 Jan 2014; 749-752 (San Francisco, CA, 2014).

7. Ahn, C. H. \& Ng, E. J. Wang VAHJHS et al. Characterization of oxide-coated polysilicon disk resonator gyroscope within a wafer-scale encapsulation process. J. Micro. Syst. 24, 1687-1694 (2015).

8. Gerrard, D. D. et al. Manipulation of heat flux paths in thermo-elastically damped resonators for Q optimization. In IEEE 30th International Conference on Micro Electro Mechanical Systems (MEMS 2017); 22-26 Jan 2017; 1130-1133 (Las Vegas, NV, 2017).

9. Nitzan, S. et al. Epitaxially-encapsulated polysilicon disk resonator gyroscope. In IEEE 26th International Conference on Micro Electro Mechanical Systems (MEMS 2013); 20-24 Jan 2013; 625-628 (Taipei, Taiwan, China, 2013).

10. Ahn, C. H. et al. Encapsulated high frequency (235 khz), high-Q (100 k) disk resonator gyroscope with electrostatic parametric pump. Appl. Phys. Lett. 105, 243504 (2014)

11. Kim, D. \& Closkey, R. M. A MEMS vibratory gyro with mode-matching achieved by resonator mass loading. In 2014 IEEE/ION Position, Location and Navigation Symposium (PLANS 2014); 5-8 May 2014; 499-503 (Monterey, CA, 2014).

12. Gerrard, D. D. et al. Q-factor optimization in disk resonator gyroscopes via geometric parameterization. In IEEE 29th International Conference on Micro Electro Mechanical Systems (MEMS 2016); 24-28 Jan 2016; 994-997 (Shanghai, 2016).

13. Zhou, X. et al. Mitigating thermoelastic dissipation of flexural micromechanical resonators by decoupling resonant frequency from thermal relaxation rate. Phys. Rev. Appl. 8, 064033 (2018).

14. Mirjalili, R. et al. Substrate-decoupled silicon disk resonators having degenerate gyroscopic modes with $\mathrm{Q}$ in excess of 1-million. In The 18th International Conference on Solid-State Sensors, Actuators and Microsystems (TRANSDUCERS 2015); 21-25 June 2015; 15-18 (Anchorage, 2015).

15. Askari, S. et al. Near-navigation grade quad mass gyroscope with Q-factor limited by thermo-elastic damping. In Solid-StateSensors, Actuators, and Microsystems Workshop; 5-9 June 2016; 254-257 (Hilton Head Island, South Carolina, 2016).

16. Cho, J. et al. Fused silica micro birthbath shell resonators with 1.2 million Q and 43 second decay time constant. In Solid-State Sensors, Actuators and Microsystems Workshop; 8-12 June 2014; 103-104 (Hilton Head Island, South Carolina, 2014).

17. Nagourney, T. et al. 259 second ring-down time and 4.45 million quality factor in 5.5khz fused silica birdbath shell resonator. In The 19th International Conference on Solid-State Sensors, Actuators and Microsystems (Transducers 2017); 18-22 June 2017; 790-793 (Kaohsiung, Taiwan, China, 2017).

18. Zhou, X. et al. Decaying Time Constant Enhanced MEMS Disk Resonator for High Precision Gyroscopic Application. IEEE/ASME Trans. Mechatron. 23, 452-458 (2018).

19. Weinberg, $\mathrm{M}$. et al. Energy loss in MEMS resonators and the impact on inertial and RF devices. In The 15th International Solid-state Sensors, Actuators and Microsystems Conference (Transducers 2009); 21-25 June 2009; 688-695 (Denver, CO, 2009)

20. Bao, M. \& Yang, H. Squeeze film air damping in MEMS. Sens. Actuators A-Phys. 136, 3-27 (2007).
21. Serrano, D. E. et al. Substrate-decoupled, bulk-acoustic wave gyroscopes: Design and evaluation of next-generation environmentally robust devices. Microsyst. \& Nanoeng. 2, 16015 (2016).

22. Prabhakar, S. \& Vengallatore, S. Theory of thermoelastic damping in micromechanical resonators with two-dimensional heat conduction. J. Micro. Syst. 17, 494-502 (2008).

23. Zhili, $\mathrm{H}$. et al. Energy loss mechanisms in a bulk-micromachined tuning fork gyroscope. In IEEE 5th Conference on Sensors (Sensors 2006) 22-25 Oct. 2006; 1333-1336 (Daegu, 2006).

24. Hossain, S. T., McWilliam, S. \& Popov, A. A. An investigation on thermoelastic damping of high-Q ring resonators. Int. J. Mech. Sci. 106, 209-219 (2016).

25. Zener, C. Internal friction in solids. I. Theory of internal friction in reeds. Phys. Rev. 52, 230-235 (1937).

26. Zener, C. Internal friction in solids II. General theory of thermoelastic internal friction. Phys. Rev. 53, 90-99 (1938).

27. Zener, C., Otis, W. \& Nuckolls, R. Internal friction in solids III. Experimental demonstration of thermoelastic internal friction. Phys. Rev. 53, 100-101 (1938).

28. Zhou, X. et al. An investigation on the ring thickness distribution of disk resonator gyroscope with high mechanical sensitivity. Int. J. Mech. Sci. 117 174-181 (2016)

29. Zhou, $X$. et al. The mechanical sensitivity optimization of a disk resonator gyroscope with mutative ring thickness. In IEEE 3th International Symposium on Inertial Sensors and Systems (IIISS 2016); 22-25 Feb 2016, 54-57 (Laguna Beach, CA, 2016).

30. Xiao, D. et al. Design of a disk resonator gyroscope with high mechanical sensitivity by optimizing the ring thickness distribution. J. Micro. Syst. 25 , 606-616 (2016)

31. Yuan, W. et al. Application of an optimization methodology for multidisciplinary system design of microgyroscopes. Microsyst. Technol. 12, 315-323 (2006).

32. Kaveh, A. et al. Structural topology optimization using ant colony methodology. Eng. Struct. 30, 2559-2565 (2008).

33. Lake, J. \& Duwel, A. E. \& Candler RN. Particle Swarm Optimization for Design of Slotted MEMS Resonators With Low Thermoelastic Dissipation. J. Micro. Syst. 23, 364-371 (2014)

34. Eberhart, R. \& Kennedy, J. A new optimizer using particle swarm theory. In The 6th International Symposium on Micro Machine and Human Science; 4-6 Oct 1995; 39-43 (Nagoya, 1995).

35. Kennedy, J. \& Eberhart, R. Particle swarm optimization. In IEEE International Conference on Neural Networks; Nov 2-Dec 1, 1995; 1942-1948 (Perth, 1995).

36. Chen, F., Li, X. \& Kraft, M. Electromechanical sigma-delta modulator $(\Sigma \Delta M)$ force feedback interfaces for capacitive MEMS inertial sensor: a review. leee. Sens. J. 16, 6476-6495 (2016).

37. Prikhodko, I. P. et al. Mode-matched MEMS coriolis vibratory gyroscopes: Myth or reality? In IEEE/ION Position, Location and Navigation Symposium (PLANS 2016); 11-14 April 2016; 1-4 (Savannah, GA, 2016).

38. Prikhodko, I. P. et al. Half-a-month stable 0.2 degree-per-hour mode-matched MEMS gyroscope. In IEEE 4th International Symposium on Inertial Sensors and Systems (Inertial 2017); 27-30 March 2017; 1-4 (Kauai, Hawaii, 2017). 